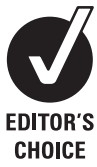

${ }^{1}$ Department of Ophthalmology, University of Minnesota, Minneapolis, Minnesota, USA;

${ }^{2}$ Department of Neurology, University of Minnesota,

Minneapolis, Minnesota, USA;

${ }^{3}$ Department of Neurosurgery, University of Minnesota,

Minneapolis, Minnesota, USA:

${ }^{4}$ Department of Otolaryngology,

University of Minnesota,

Minneapolis, Minnesota, USA

${ }^{5}$ Department of Ophthalmology,

Emory University, Atlanta,

Georgia, USA

Correspondence to:

Dr M S Lee, 420 Delaware

Street SE, MMC 493,

Minneapolis, MN 55455, USA;

mikelee@umn.edu

Accepted 22 August 2008

Published Online First

1 October 2008

\title{
Hydroxychloroquine retinopathy screening
}

\author{
A E Semmer, ${ }^{1}$ M S Lee, ${ }^{1,2,3}$ A R Harrison, ${ }^{1,4}$ T W Olsen ${ }^{5}$
}

\section{ABSTRACT}

Aim: To compare current hydroxychloroquine retinopathy screening practices with the published 2002 American Academy of Ophthalmology (AAO) Preferred Practice Patterns (PPP).

Methods: A multiple-choice survey was distributed to 105 ophthalmologists to assess current screening practices and knowledge of patient risk factors. Results were compared with the PPP guidelines. A cost analysis of the PPP and survey paradigms was conducted.

Results: Sixty-seven (64\%) of 105 surveys were completed. The majority (90\%) of physicians screen for hydroxychloroquine retinopathy with either central automated threshold perimetry or Amsler grid as recommended by the PPP. Most survey respondents could not correctly identify the evidence-based risk factors. The majority screen more frequently than recommended: $87 \%$ screen high-risk patients and $94 \%$ screen low-risk patients more frequently than recommended in the PPP. The increased screening frequency of low-risk patients translates into an excess of $\$ 44$ million in the first 5 years of therapy. If all patients were screened using exact PPP paradigm, savings could exceed $\$ 150$ million every 10 years.

Conclusions: Ophthalmologists currently screen for hydroxychloroquine retinopathy correctly; however, their lack of familiarity with evidence-based guidelines may result in excessive follow-up. Increasing awareness and implementation of the PPP could potentially reduce hydroxychloroquine retinopathy screening costs significantly.

Since the 1950s, antimalarial drugs have been used to treat various autoimmune diseases including systemic lupus erythematosus and rheumatoid arthritis. In the United States, hydroxychloroquine is the antimalarial drug of choice because of its low retinal toxicity. ${ }^{1-4}$ Between 1960 and 2005, only 47 cases of hydroxychloroquine retinopathy have been reported in the peer-reviewed literature. ${ }^{5}$ Despite the existence of unreported cases, hydroxychloroquine retinopathy is rare. The exact pathophysiology remains unknown, but daily dose, duration of therapy, renal function, liver function and patient age modify patient risk. ${ }^{1-5}$

The ophthalmology and rheumatology literature continually debate the most appropriate paradigm for hydroxychloroquine retinopathy screening. ${ }^{24-7}$ 12-14 In 2002, the American Academy of Ophthalmology (AAO) addressed this controversy by publishing preferred practice patterns (PPP) for hydroxychloroquine retinopathy screening. These evidence-based guidelines attempted to maximise practicality and optimise the cost/benefit ratio of hydroxychloroquine retinopathy screening. They were designed as guidelines that physicians might choose to modify based on their clinical judgement, patient preference and medicolegal concerns. ${ }^{7}$

The PPP recommends baseline examination and risk assessment within the first year of therapy. There is no need for follow-up in the first 5 years for low-risk patients; however, the PPP recommend annual screening of high-risk patients. High-risk patients are those with: (1) daily hydroxychloroquine exceeding $6.5 \mathrm{mg} / \mathrm{kg}$, (2) duration of therapy greater than 5 years, (3) age greater than 60, (4) obesity, (5) renal disease, (6) hepatic disease or (7) concurrent retinal disease. ${ }^{8}$

The PPP hydroxychloroquine retinopathy screening exams include a comprehensive ophthalmological evaluation with central visual-field assessment by either Amsler grid or Humphrey Visual Field (HVF) 10-2 perimetry (Zeiss, Dublin, CA). Colour vision testing, fundus photography, fluorescein angiography and multifocal electroretinography (mfERG) are considered optional. ${ }^{8}$

This investigation compared the current hydroxychloroquine screening practices of community ophthalmologists to the guidelines outlined in the PPP. Knowledge of risk factors and recommended follow-up frequencies were assessed along with the financial implications of current screening methods.

\section{MATERIALS AND METHODS}

An anonymous, nine question, multiple-choice survey was distributed to 105 attendees of the 2007 Twin Cities Eye Meeting prior to a hydroxychloroquine presentation. The survey focused on physician's hydroxychloroquine screening practices and their knowledge of risk factors for developing hydroxychloroquine retinopathy. Following institutional review board approval, survey results were compared with the PPP guidelines.

Based on the survey results, we performed a conservative cost analysis comparing current screening costs to the hypothetical cost of screening all patients according to the PPP recommended protocol. Although the PPP recommends only one initial visit for low-risk patients in their first 5 years of therapy, we accounted for the cost of additional routine AAO-recommended ophthalmological exams that may occur during this time for certain age groups (table 1). ${ }^{9}$

Since hydroxychloroquine patient demographics are not known, we assumed that 150000 patients are taking hydroxychloroquine at any time in the United States. ${ }^{2}$ The age distribution of these patients was estimated from hydroxychloroquine patient ages published in two previous studies (25\% under age $40,31 \%$ ages $40-54,12 \%$ ages 55 60 and $33 \%$ over age 60). ${ }^{11}$ These ages were then projected onto our estimated population of 150000 patients. 
We arbitrarily assumed that 10-20\% of patients under age 60 (7-15\% of the total patient population) are high-risk for hydroxychloroquine retinopathy due to one or more of the following: daily dose greater than $6.5 \mathrm{mg} / \mathrm{kg}$, duration of therapy greater than 5 years, obesity or underlying liver, kidney or retinal disease. Combining these patients with those over age $60(33 \%)$ who are high-risk based solely on age, it follows that approximately $40-50 \%$ of the estimated 150000 US hydroxychloroquine patients are at high risk for developing retinopathy. The remaining $50-60 \%$ of patients are considered low-risk.

Screening costs were estimated at $\$ 108$ per exam based on Medicare reimbursement of a Level 3 eye exam (\$35) and HVF testing (\$73). The cost of optional screening exams was not included.

\section{RESULTS}

Sixty-seven (64\%) of the 105 distributed surveys were completed. The majority of respondents (67\%) were comprehensive ophthalmologists, but all ophthalmological subspecialties were represented at least once. The majority (55\%) of ophthalmologists screen between one and 25 hydroxychloroquine patients a year. Of the remainder, $31 \%$ screen $26-50,12 \%$ screen $51-75$, and $2 \%$ screen $76-100$ patients annually.

\section{Screening techniques}

The vast majority (90\%) of the surveyed ophthalmologists screen hydroxychloroquine patient's visual fields as recommended in the PPP. Fifty-four (81\%) use macular automated perimetry, seven $(10 \%)$ combine perimetry with Amsler grid and six (9\%) use Amsler grid alone. In addition, many physicians perform optional exams including colour vision tests $(73 \%)$ and fundus photography (28\%). Less common exams were fluorescein angiography (3\%) and electroretinography (ERG) (3\%). None of the participants screen with the mfERG.

\section{Knowledge of risk factors}

Knowledge of the evidence-based quantitative risk factors (daily dose, duration of therapy, patient age) was poor. As shown in table 2, the majority of survey respondents failed to identify the risk factors. Few correct responses were given, and no respondent identified all three risk factors. Twenty-six (39\%) responded "I don't know" to all three risk-factor questions. On average, the number of "I don't know" responses was similar for all ophthalmologists, regardless of the annual hydroxychloroquine patients seen.

\section{Follow-up}

While all ophthalmologists appropriately screen high-risk patients more often than low-risk patients, most screen all of their patients more frequently than recommended in the PPP. Eighty per cent screen high-risk patients every 6 months, which is twice as frequent as the PPP recommendation. Sixty-five per

Table 1 American Academy of Ophthalmology routine comprehensive exam recommendations

\begin{tabular}{ll}
\hline Age (years) & Exam frequency (years) \\
\hline$<40$ & $05-10$ \\
$40-54$ & $02-4$ \\
$55-64$ & $01-3$ \\
$65+$ & $01-2$ \\
\hline
\end{tabular}

Adapted from the AAO Comprehensive Adult Medical Eye Evaluation Preferred Practice Pattern. ${ }^{9}$ cent screen low-risk patients annually. The PPP recommends only one initial visit for these patients in the first 5 years.

\section{Cost analysis}

Cost analysis revealed significant potential savings if current screening were modified to fit the PPP paradigm. Using conservative cost estimates, screening all low-risk patients in the first 5 years of therapy currently costs approximately $\$ 56.8$ million. In contrast, we estimated it would cost $\$ 12.9$ million for the first 5 years of therapy if low-risk patients were screened strictly according to the PPP. These estimates suggest a potential savings of $\$ 44$ million every 5 years if all physicians would screen their low-risk patients without deviating from the PPP.

Current high-risk patient screening was estimated to cost $\$ 68.9$ million every 5 years, while the PPP paradigm would cost approximately $\$ 36.4$ million. This translates into an additional \$32.4 million 5-year potential savings if high-risk patients were screened strictly using the PPP paradigm.

If we assume the hydroxychloroquine patient population size and demographics are constant over time, these estimates suggest a potential savings of $\$ 152.6$ million every 10 years if all patients were screened precisely according to the PPP recommendations.

\section{DISCUSSION}

We found that community ophthalmologists generally screen for hydroxychloroquine retinopathy as recommended in the PPP. In addition to comprehensive eye exams, $90 \%$ of entire group regularly test visual fields with Amsler grid or automated central threshold perimetry.

Although this overall screening competency is reassuring, the general lack of familiarity with evidence-based guidelines may affect patient care and healthcare costs.

We estimated 10-year savings of over $\$ 150$ million using the evidence-based guidelines for all patients. We acknowledge that high-risk patients may necessitate more frequent screening based on both physician and patient concern. However, it seems reasonable to follow the PPP for low-risk patients. Therefore, the most significant potential savings exist in low-risk hydroxychloroquine retinopathy screening.

Table 2 Knowledge of American Academy of Ophthalmology defined risk factors

\begin{tabular}{lr}
\hline & No of responses (\%) \\
\hline Dose $>6.5 \mathrm{mg} / \mathrm{kg}$ & \\
Correct & $9(13)$ \\
Incorrect & $58(87)$ \\
Overestimate & $9(16)$ \\
Underestimate & $11(19)$ \\
$\quad$ Unsure & $38(66)$ \\
Duration $>5$ years & \\
Correct & $18(27)$ \\
Incorrect & $49(73)$ \\
Overestimate & $8(16)$ \\
Underestimate & $9(18)$ \\
$\quad$ Unsure & $32(65)$ \\
Age $>60$ years & \\
Correct & $7(10)$ \\
Incorrect & $60(90)$ \\
Overestimate & $1(1)$ \\
Underestimate & $14(23)$ \\
Unsure & $45(75)$ \\
\hline
\end{tabular}


Our estimated $\$ 44$ million spending excess in the first 5 years of therapy for low-risk hydroxychloroquine patient screening in the United States does not include the cost of fundus photography, fluorescein angiography or electrophysiology. It also does not include the economic impact of missed work, transportation, parking, copayments, and childcare for patient visits. Therefore, the actual economic impact of current low-risk hydroxychloroquine retinopathy screening is likely much greater than our conservative estimate of $\$ 88$ million over 10 years.

While some of this cost excess is justified by more frequent follow-up with select patients, screening most low-risk patients more than recommended leads to inconvenience and excessive healthcare costs. Strict implementation of the evidence-based guidelines into clinical practice, with modification on an individual case basis when necessary, could potentially increase the cost-effectiveness of care. Moreover, increased use of lowcost self-testing and education (Amsler Grid) could be better utilised.

The survey respondents' lack of knowledge regarding evidence-based follow-up intervals suggests physicians may not consciously deviate from the PPP guidelines, but instead are less familiar with current screening standards. Interestingly, we found that nearly half (49\%) of the survey respondents follow up at 6 months/12 months for high- and low-risk patients, respectively. This may represent a persistent screening protocol presumably acquired prior to the 2002 PPP publication. This suggests that the hydroxychloroquine screening cost/benefit ratio may be optimised simply by increasing awareness of the PPP recommendations and encouraging physicians to incorporate them into practice.

One major limitation of this study is that the opinions and practices of 67 individuals are assumed to represent all US ophthalmologists. This sample size is not ideal; however, substantial trends were seen that suggest a widespread, inadequate knowledge base among ophthalmologists. While a larger-scale survey would give a better representation of US screening practices, simply raising physician awareness of the hydroxychloroquine retinopathy PPP may obviate the need for further investigation. Another potential criticism is that the majority (55\%) of survey respondents perform between one and 25 screening exams a year. Although no direct correlation was seen between familiarity with hydroxychloroquine retinopathy risk factors and increased patient load, knowledge may vary greatly between physicians at the extremes of each range. A more finite range may add insight to the lack of physician familiarity with the PPP.

An additional potential weakness of this study is that multiple assumptions were needed to estimate hydroxychloroquine patient demographics, especially the number of high-risk patients under 60 years old. However, our patient risk stratification is comparable with a recent study of 109 hydroxychloroquine patients who were screened at the San Francisco Veterans Affairs Medical Center. In this study, 32 $(29 \%)$ of the patients under 60 years old were high-risk. ${ }^{15}$ In our study, we assumed that $10-20 \%$ of the patients under 60 were high-risk based on the same criteria. While our estimate is smaller, it accounts for a more diverse patient population that is not limited to a VA patient population.
Ultimately, the AAO will eventually re-evaluate their hydroxychloroquine retinopathy screening recommendations in light of the evolving technology. Recent studies suggest that mfERG may represent the future of hydroxychloroquine retinopathy screening. ${ }^{81-14}$ Its objective analysis of the parafoveal retina may prove to be the most effective means of detecting possibly reversible, early retinopathy. While this is an exciting development in ophthalmological care, current mfERG use is not widespread, and its future role in hydroxychloroquine retinopathy screening is not fully understood. It seems unlikely that mfERG will replace visual-field testing, and adding mfERG to screening protocols may result in greater overall costs.

The incidence of hydroxychloroquine retinopathy is low, but its consequences can be devastating. Together, physicians and patients must decide if the benefits of hydroxychloroquine outweigh its risks. While the PPP were intended as patient care guidelines, they represent an evidence-based balance of the cost/ benefit ratio and deserve consideration by all screening clinicians. Until new guidelines are developed, we must rely on the evidence-based screening practices outlined in the PPP to guide our minimum standard of care. While we await new recommendations, a national effort to increase awareness and implementation of the PPP would be simple and inexpensive, and may significantly reduce the economic impact of hydroxychloroquine retinopathy screening.

Funding: This work was supported by an unrestricted grant to the University of Minnesota from Research to Prevent Blindness New York and Minnesota Lions.

Competing interests: None.

Ethics approval: Ethics approval was provided by the Institutional Review Board, University of Minnesota, Minneapolis, MN.

\section{REFERENCES}

1. Hobbs H, Sorsby A, Freedman A. Retinopathy following chloroquine therapy. Lancet 1959;2:478-80.

2. Levy GC, Munz SJ, Paschal J, et al. Incidence of hydroxychloroquine retinopathy in 1,207 patients in a large multicenter outpatient practice. Arthritis Rheum 1997:40:1482-6.

3. Finbloom DS, Silver K, Newsome DA, et al. Comparison of hydroxychloroquine and chloroquine use and the development of retinal toxicity. J Rheumatol 1985;12:692-4.

4. Bernstein HN. Ocular safety of hydroxychloroquine. Ann Ophthalmol 1991;23:2926.

5. Yam JC, Kwok AK. Ocular toxicity of hydroxychloroquine. Hong Kong Med J 2006;12:294-304.

6. Easterbrook M. Screening for antimalarial toxicity. Can J Ophthalmol 1993;28:51-2.

7. Ruiz RS, Saatci OA. Chloroquine and hydroxychloroquine retinopathy: how to follow affected patients. Ann Ophthalmol 1991;23:290-1.

8. Marmor MF, Carr RE, Easterbrook M, et al. Recommendations on screening for chloroquine and hydroxychloroquine retinopathy: a report by the American Academy of Ophthalmology. Ophthalmology 2002;109:1377-82.

9. American Academy of Ophthalmology. Comprehensive adult medical eye evaluation, preferred practice pattern. San Francisco: American Academy of Ophthalmology, 2005. http://www.aao.org/ppp (accessed 5 Oct 2008).

10. Grierson DJ. Hydroxychloroquine and visual screening in a rheumatology outpatient clinic. Ann Rheum Dis 1997;56:188-90.

11. Elder M, Rahman AM, McLay J. Early paracentral visual field loss in patients taking hydroxychloroquine. Arch Ophthalmol 2006;124:1729-33.

12. Lai TY, Chan WM, Li H, et al. Multifocal electroretinographic changes in patients receiving hydroxychloroquine therapy. Am J Ophthalmol 2005;140:794-807.

13. Lyons JS, Severns ML. Detection of early hydroxychloroquine retinal toxicity enhanced by ring ratio analysis of multifocal electroretinography. Am J Ophthalmol 2007; 143:801-9

14. Marmor MF. The dilemma of hydroxychloroquine screening: new information from the multifocal ERG. Am J Ophthalmol 2005;140:894-5.

15. Flach AJ. Improving the risk-benefit relationship and informed consent for patients treated with hydroxychloroquine. Trans Am Ophthalmol Soc 2007;105:191-7. 